\title{
Uterine leiomyoma and its association with menstrual pattern and history of depo-medroxyprogesterone acetate injections
}

This article was published in the following Dove Press journal:

International Journal of General Medicine

I 3 July 201 I

Number of times this article has been viewed

\section{Amanti ${ }^{2}$ \\ H Sadeghi-Bazargani' \\ $\mathrm{H} \mathrm{Abdollahi}^{2}$ \\ F Ehdaeivand $^{3}$}

'Statistics and Epidemiology Department, Faculty of Health and Nutrition, Tabriz University of Medical Sciences, Tabriz, Iran; ${ }^{2}$ Tabriz University of Medical Sciences, Tabriz, Iran; ${ }^{3}$ Faculty of Medicine, Ardabil University of Medical Sciences, Ardabil, Iran
Correspondence: Hossein Mashadi Abdollahi

Tabriz University of Medical Sciences, Golgasht Street, Tabriz, Iran

Tel +989l44000823

Email h.abdollahimashadi@yahoo.com
Background and aim: Despite the high prevalence of uterine leiomyoma, according to recent review studies there is uncertainty and a paucity of information regarding its predisposing or protective factors. The aim of this study was to assess the possible association between menstrual cycle pattern and occurrence of surgically treated myomas and also to check if depomedroxyprogesterone acetate (DMPA) injection earlier in reproductive life can affect the later occurrence of myomas needing surgical treatment.

Methods: In a case-control study in Ardabil, 85 women with definite diagnosis of surgically treated uterine leiomyoma and 154 community controls were enrolled. Possible predictors of myoma including menstrual cycle and menstrual bleeding patterns were assessed. Data were analyzed using SPSS software (SPSS, IBM, Somers, NY). Odds ratios were used as the main statistic in assessing the strength of observed associations.

Results: Mean age of the participants was $41.8 \pm 8.5$ years. Length of menstrual cycle was associated with myoma and a higher likelihood of myoma was observed among those having shorter menstrual cycles $(P<0.05)$. Number of menstrual bleeding days was also associated with surgically treated myoma and longer bleeding periods increased the likelihood of myoma $(P<0.05)$. Only one of the eight women who had a history of depo-medroxyprogesterone acetate injections had developed surgically treated uterine leiomyoma and the others belonged to the control group without a history of surgical treatment for uterine leiomyoma.

Conclusion: Menstrual cycle pattern is associated with developing leiomyomas requiring surgical treatment. DMPA, other than its role in myoma treatment, is also assumed to have a role in preventing myomas, but due to the small sample size in this study, larger scale prospective trials are needed in the future.

Keywords: myoma, uterine leiomyoma, DMPA, medroxyprogesterone, menstrual cycle, menstrual, depo-provera

\section{Introduction}

Uterine leiomyoma is the most common benign tumor of the uterus, and the most common female genital neoplasm. It affects $30 \%$ of all women of reproductive age. ${ }^{1,2}$ Although uterine leiomyoma is a very common condition it has not received adequate attention from researchers, possibly due to being benign. However, uterine leiomyoma research has been underfunded even compared with other nonmalignant diseases. This may be because it is usually an asymptomatic condition. ${ }^{3}$ The lack of research attention to uterine leiomyomas affects all aspects of the condition, but this is more profound regarding etiologic studies rather than therapeutic research. There is some evidence regarding its etiology but the literature regarding uterine leiomyoma risk factors should be interpreted with caution. This is due to the paucity 
of studies available, the heterogeneity of populations, and the conflicting results, which suggest that other unexamined factors may be involved. ${ }^{2}$ Several predisposing factors or predictors of uterine leiomyoma have been identified, including age, endogenic and exogenic hormonal factors, AfricanAmerican ethnicity, nulliparity, diet, smoking, and obesity. ${ }^{2,4}$ Hormonal factors or hormone-related factors have been controversially discussed in some cases but insufficient evidence is available to support any scientific conclusions. Two main hormone-related predictors that have not been assessed for their role in predicting myomas are menstrual pattern and use of depo-medroxyprogesterone- acetate(DMPA). ${ }^{4-8}$ The use of DMPA as an FDA-approved contraceptive is too recent to expect adequate research on its predictive role for uterine leiomyoma. However, the paucity of evidence on the role of menstrual pattern in predicting myomas is attributable to the neglect of this area of reproductive health research, as discussed above. DPMA, due to its menstrual side effects, is not popular as a long-term contraceptive, but it has been used quite widely over a period of time in Iran, particularly in Ardabil province. ${ }^{9}$

The aim of this study was to assess whether menstrual pattern can predict the occurrence of surgically treated uterine myomas. A secondary objective in this research was to check whether DMPA injection earlier in reproductive life influences the occurrence of surgically treated myomas.

\section{Methods}

In a case-control study in Ardabil in 2006, 85 women with definite diagnosis of surgically treated uterine leiomyoma, along with 154 community controls, were enrolled. Cases were surgically treated leiomyomas undergoing surgery in Ardabil medical centers, with a proven pathology of leiomyoma. Cases were identified by reviewing laboratory and hospital records from medical centers in Ardabil. A questionnaire assessing possible predictors of uterine leiomyomas, including menstrual cycle and menstrual bleeding pattern, was completed for all participants. The addresses of patients were obtained, and their health and contraceptive records, available in each of the 30 primary health care centers in Ardabil, were reviewed. About double the number of controls was identified for the number of cases in each center, half of them being neighbor controls and others chosen based on a random sample of the eligible population covered by each center. Controls included healthy women lacking a known history of uterine leiomyoma or diseases related to leiomyoma.

Data were entered into the computer and analyzed using the SPSS statistical software package (v 13; SPSS IBM,
Somers, NY). Odds ratios were used as the main statistic in assessing the associations.

The study protocol was approved by the committee of ethics at Ardabil University of Medical Sciences. In both groups, informed consent was obtained before enrollment into the study.

\section{Results}

Mean age of the participants was $41.8( \pm 8.5)$ years. Thirtytwo of the participants were rural residents. No association was explored between myoma and background variables including literacy, occupation, age at first marriage, age at first delivery, and age at last delivery. Parity, gravidity, history of abortion, and history of cesarean section didn't have a statistically significant difference between the case and control groups. Comparison of some background variables between case and control group participants is given in Table 1.

The median number of myomas was one lesion per participant, but some had multiple myomas. The largest myoma was $127 \times 116 \times 90 \mathrm{~mm}$ in size. Nearly two-thirds of the surgeries were hysterectomies while the remainder had undergone myomectomies.

The mean length of menstrual cycle among all participants was $27.9 \pm 5.9$ days. Mean menstruation bleeding length was $6.1 \pm 3.6$ days. Mean length of menstrual cycle among cases and controls were $26.7 \pm 5$ and $28.5 \pm 6.4$ days respectively. Mean menstruation bleeding length was $6.9 \pm 5.4$ days for the case group and 5.6 \pm 3.6 days for the control group, respectively. Length of menstrual cycle was associated with myoma and a higher likelihood of myoma was observed among those having shorter menstrual cycles $(P<0.05)$. Number of menstrual bleeding days was also associated with surgically treated myoma and a longer bleeding period increased the likelihood of myoma $(P<0.05)$. As an exploratory bivariate

Table I Comparison of some background variables between case and control group participants

\begin{tabular}{|c|c|c|c|}
\hline & Case & Control & $\begin{array}{l}\text { Statistical } \\
\text { significance }\end{array}$ \\
\hline $\begin{array}{l}\text { Mean years of } \\
\text { successful education }\end{array}$ & 6.64 & 5.59 & NS \\
\hline $\begin{array}{l}\text { Mean age at first } \\
\text { delivery }\end{array}$ & 19.2 & 18.8 & NS \\
\hline $\begin{array}{l}\text { Mean age at last } \\
\text { delivery }\end{array}$ & 30.3 & 31.2 & NS \\
\hline $\begin{array}{l}\text { Mean age at first } \\
\text { marriage }\end{array}$ & 17.5 & 16.8 & NS \\
\hline Mean gravidity & 5 & 5.2 & NS \\
\hline Mean parity & 4.5 & 4.6 & NS \\
\hline
\end{tabular}

Abbreviation: NS, No statistically significant difference. 
analysis, the odds ratios of myoma for different cutoff levels of menstrual cycle length are given in Table 2 along with their $95 \%$ confidence intervals.

Myoma was more likely among menopausal women $(P<0.01)$. Among myoma cases, $18.5 \%$ were menopausal while this figure was $3.5 \%$ for the control group. A history of infertility, tubectomy, number of live births, and time after last delivery were not found to be associated with myoma. History of menometrorrhagia increased the odds of myoma by 3.1 times (95\% CI: 1.5-6.8).

Among the participants, eight women had used DMPA injection as a contraceptive during the 10 years before the interview. Only one case of these had developed myoma and the others belonged to the control group without surgically treated myomas. The low expected count violated the assumption to use chi-square test and Fisher's exact test didn't show the difference to be statistically significant. Socioeconomic level and urbanization were not found to be associated with higher likelihood of myoma.

\section{Discussion}

The association between menstrual pattern and myoma has been found to be inconsistent in the literature. Chen et al, in a large case-control study, found a menstrual cycle length of $>30$ days (OR: 1.6 ; 95\% CI: 1.1-3.3), and menstrual bleeding for $\geq 6$ days (OR: $1.4 ; 95 \% \mathrm{CI}: 1.0-2.0$ ) to be associated with developing myoma ${ }^{8}$ In contrast, another study enrolling 73 women with myoma diagnosed after ultrasonography done on 341 Italian premenopausal women, didn't find such an association. ${ }^{7}$ One main similarity between the study by Chen and ours was that they enrolled patients from those undergoing tubal ligation, and controls were selected from among women with no laparoscopic evidence of the history of fibroids. Similarly we also didn't rule out the existence of possibly mild forms of myoma in the control group by ultrasonography. However, in the Italian study the outcome of interest was myoma diagnosed during screening by ultrasonography. So one theory explaining the contrast in

Table 2 Odds ratios for different cutoffs of menstrual cycle lengths for surgically treated myoma, and related confidence intervals

\begin{tabular}{lll}
\hline $\begin{array}{l}\text { Menstrual } \\
\text { cycle length }\end{array}$ & Odds ratio & $\begin{array}{l}\mathbf{9 5} \% \text { Confidence } \\
\text { interval }\end{array}$ \\
\hline$<22$ days & $3 / 8$ & $1 / 34-1 \mathrm{I} / 08$ \\
$<23$ days & $3 / 3$ & $1 / 21-9 / 19$ \\
$<24$ days & $3 / 18$ & $1 / 8-8 / 44$ \\
$<25$ days & $2 / 42$ & $1 / 23-4 / 7$ \\
$<26$ days & $1 / 85$ & $0 / 97-3 / 55$ \\
\hline
\end{tabular}

Italian study is that menstrual pattern may not be associated with developing myoma but it may only explain symptomatic or larger myomas. This needs to be further clarified in future focused studies. Other than similarities in methodology and results of our study and that of Chen et $\mathrm{al}^{8}$ some differences also existed and need to be considered in interpreting the findings of the two studies. To prevent an ascertainment bias we limited our enrollment to surgically removed myomas. The age range in Chen's study was limited to $17-44$ years. Ours lacked such a limitation but our patients were older on average. We didn't report age as a predictor because as age is a known factor for myoma, we preferred to match it in order to increase the statistical power of our analysis.

It seems that the association between length of menstrual cycle and myoma may not be linear. Chen et $\mathrm{al}^{8}$ explored the effect of longer cycles while our study looked at the role of shorter cycles, as in our study very few women were found with longer cycle lengths. Regarding the association found between menometrorrhagia and myoma in our study it should be taken into account that it can be interpreted as a symptom of myoma rather than a risk factor, because the temporality between these two conditions cannot be well clarified in a case-control study, considering the disease induction period and recall bias. In line with the findings of Wise and Chen, we didn't find an association between myoma and use of oral contraceptive pills (OCPs). ${ }^{6,8}$ Contrary to Chen, the study by Wise et al, ${ }^{6}$ and our own findings, some studies have indicated that OCPs have a preventive role in uterine myoma. ${ }^{5,10-12}$ However, Ross et al found such an effect only after at least 10 years of OCP use. ${ }^{10}$ Interestingly, contrary to these studies, one study from Iran ${ }^{13}$ has found that OCP use may increase the likelihood of uterine myomas, although it seems that this finding may be due to ascertainment and selection bias as it studied self-reported myomas. Depo-medroxyprogesterone acetate (DMPA) was found to be more frequent in the myoma group, but this was not statistically significant. The authors consider the lack of statistical significance to be due to the low statistical power of the study. Because DMPA is not a long-established FDA-approved drug for contraceptive use, and as those in our study were generally older, the overall frequency of DMPA use was low among our participants. Nevertheless, if this tendency becomes significant in larger scaled studies, a possible mechanism may be assumed based on the theory of estrogen dependence of uterine leiomyomas. The observed protective effect of DMPA against myomas may be explained by the hypo-estrogenic state induced by DMPA usage or by endometrial suppression induced by longer term use of DMPA. $., 14,15$ 


\section{Limitations}

Surgically treated myoma was selected as the outcome in this study to prevent ascertainment bias and to discriminate between cases and controls with lower cost and effort. Otherwise, due to the high prevalence of subclinical myomas, the study would have been both expensive and more prone to bias. However, it should be taken into account that limiting the study to surgically treated myomas decreases the generalizability and limits drawing stronger conclusions. If a preventive factor is explored it may be due to the role of that factor in slowing the disease process rather than preventing it: in other words, it prevents the development of more severe myoma cases. This should be considered when interpreting the results of this study or other similar studies.

\section{Conclusion}

Menstrual cycle pattern is associated with the development of leiomyomas requiring surgical treatment. DMPA is assumed to have a role in preventing myomas, but due to the small sample size in this study, prospective trials are needed in the future.

\section{Disclosure}

The authors report no conflicts of interest with this research.

\section{References}

1. Parker WH. Uterine myomas: an overview of development, clinical features, and management. Obstet Gynecol. 2005;105(1):216-217.

2. Parker WH. Etiology, symptomatology, and diagnosis of uterine myomas. Fertil Steril. 2007;87(4):725-736.

3. Walker CL, Stewart EA. Uterine fibroids: the elephant in the room. Science. 2005;308(5728):1589-1592.
4. El-Shennawy GA, Elbialy AA, Isamil AE, El Behery MM. Is genetic polymorphism of ER-alpha, CYP1A1, and CYP1B1 a risk factor for uterine leiomyoma? Arch Gynecol Obstet. 2011;283(6):1313-1318.

5. Lumbiganon P, Rugpao S, Phandhu-fung S, Laopaiboon M, Vudhikamraksa N, Werawatakul Y. Protective effect of depotmedroxyprogesterone acetate on surgically treated uterine leiomyomas: a multicentre case-control study. Br J Obstet Gynaecol. 1996;103(9): 909-914.

6. Wise LA, Palmer JR, Harlow BL et al. Reproductive factors, hormonal contraception, and risk of uterine leiomyomata in African-American women: a prospective study. Am J Epidemiol. 2004;159(2):113-123.

7. Marino JL, Eskenazi B, Warner M, et al. Uterine leiomyoma and menstrual cycle characteristics in a population-based cohort study. Hum Reprod. 2004;19(10):2350-2355.

8. Chen CR, Buck GM, Courey NG, Perez KM, Wactawski-Wende J. Risk factors for uterine fibroids among women undergoing tubal sterilization. Am J Epidemiol. 2001;153(1):20-26.

9. Sadeghi-Bazargani H, Ehdaeivand F, Arshi S, Eftekhar H, Sezavar H, Amanati L. Low-dose oral contraceptive to re-induce menstrual bleeding in amenorrheic women on DMPA treatment: a randomized clinical trial. Med Sci Monit. 2006;12(10):CR420-CR425.

10. Ross RK, Pike MC, Vessey MP, Bull D, Yeates D, Casagrande JT. Risk factors for uterine fibroids: reduced risk associated with oral contraceptives. Br Med J (Clin Res Ed). 1986;293(6543):359-362.

11. Faerstein E, Szklo M, Rosenshein N. Risk factors for uterine leiomyoma: a practice-based case-control study. I. African-American heritage, reproductive history, body size, and smoking. Am J Epidemiol. 2001;153(1):1-10.

12. Faerstein E, Szklo M, Rosenshein NB. Risk factors for uterine leiomyoma: a practice-based case-control study. II. Atherogenic risk factors and potential sources of uterine irritation. Am J Epidemiol. 2001; 153(1):11-19.

13. Samadi AR, Lee NC, Flanders WD, Boring JR, III, Parris EB. Risk factors for self-reported uterine fibroids: a case-control study. Am J Public Health. 1996;86(6):858-862.

14. Bassaw K, Gangar K. Protective effect of depot-medroxyprogesterone acetate on surgically treated uterine leiomyomas: a multicentre casecontrol study. Br J Obstet Gynaecol. 1997;104(6):758-759.

15. Ikomi AA, Singer A. Protective effect of depot-medroxyprogesterone acetate on surgically treated uterine leiomyomas: a multicentre casecontrol study. Br J Obstet Gynaecol. 1997;104(3):385-386.
International Journal of General Medicine

\section{Publish your work in this journal}

The International Journal of General Medicine is an international, peer-reviewed open-access journal that focuses on general and internal medicine, pathogenesis, epidemiology, diagnosis, monitoring and treatment protocols. The journal is characterized by the rapid reporting of reviews, original research and clinical studies across all disease areas.

\section{Dovepress}

A key focus is the elucidation of disease processes and management protocols resulting in improved outcomes for the patient.The manuscript management system is completely online and includes a very quick and fair peer-review system. Visit http://www.dovepress.com/ testimonials.php to read real quotes from published authors. 